\title{
Reliable Software Systems Design: Defect Prevention, Detection, and Containment
}

\author{
Gerard J. Holzmann and Rajeev Joshi \\ Laboratory for Reliable Software (LaRS) \\ Jet Propulsion Laboratory, California Institute of Technology \\ Pasadena, CA 91109, USA
}

\begin{abstract}
The grand challenge that is the focus of this conference targets the development of a practical methodology for software verification: a methodology that can help us to reduce the number of residual defects in software products. Reducing residual defects is of course not in itself the objective of this exercise; the true objective is to reduce the number of failures in the use of software products. Or in other words: the objective is the development of a methodology for "reliable software systems design."
\end{abstract}

\section{Introduction}

It has often been argued that with the right training, discipline, and tools it should be possible to produce zero-defect code. Very few things in life, though, are zero-defect - not even the things that can be considered life critical. If you practice sky-diving, you are probably acutely aware that your main parachute could fail to open, no matter how carefully you check it before each jump. The parachutist would also be wise not to trust a company that tries to sell him a zero-defect parachute. He is more likely to avoid disaster by bringing a spare chute on every jump. That is: the seasoned parachutist takes the possibility of component failure into account to achieve a lowered probability of system failure. Elevators are another good example. Of course, any elevator component can fail, including the cable from which the elevator cab is suspended. But, the elevator system as a whole is designed in such a way that even when the cable breaks, the car will not come crashing down. We trust the system, even though we know that none of its components are zero-defect. For the parachute redundancy can trivially be used, but in the case of the elevator redundancy does not necessarily solve the problem. Multiple cables may help address one specific form of component failure, but operating multiple elevators in parallel would not address the real safety issue that is at stake here.

Reliable systems are always designed with the possibility of component failure in mind, and with remedies in place to significantly reduce the odds of system failure.

It is worth contemplating how deeply engrained the discipline of reliable system design is, outside software engineering. If your kitchen-sink leaks, you can close a valve that stops the flow of water to that sink. The valve is there because experience has shown that sinks do occasionally leak, no matter how carefully they are constructed to prevent just that. If you short-circuit an electrical outlet in your home, a 
fuse will blow. The fuse is there to prevent greater disaster in case the unimaginable happens. The presence of the fuse and the valve do not signify an implicit acceptance of sloppy workmanship; they are an essential part of reliable system design.

In contrast, most software today is build without valves and fuses. We try to build perfect parachutes that do not need a backup. When software fails, we blame the developer for failing to be perfect. Would it not be wiser to assume from the start that even carefully constructed and verified software components, like all other things in life, may fail in unexpected ways, and use this knowledge to construct assemblies of software components that provide independently verifiable system reliability?

\section{Building Reliable Systems from Unreliable Parts}

Hardware designers already know how to construct reliable systems from unreliable parts. In building these systems, the designer starts from the knowledge that any component in the system might fail, while securing that such failures can not cause the failure of the system as a whole. We have yet to learn how to apply similar principles in the construction of reliable software systems.

Any improvement in this domain will have to be grounded firmly in strong software verification techniques, some of which exist and some of which remain to be developed. The purpose of this position paper, though, is to point out that the development of those techniques alone will not suffice. Our ultimate objective, after all, is not necessarily to produce zero-defect software, but to produce ultra-reliable software systems. This position has implications for the type of work we need to do, as we will outline in more detail in the remainder of this paper.

\subsection{Blue Screens of Death}

Non-critical software applications are often designed in a monolithic fashion. When the application crashes, e.g. when it hits a divide by zero error, the only recourse one then has is to restart the application from scratch. This approach is, of course, not adequate to use in the construction of systems that are safety critical, for instance because human life depends on its correct and continued functioning. When, for instance, a spacecraft experiences an unexpected failure of one of its components during a launch or landing procedure, a complete restart of the software may in itself cost the loss of the mission. In manned space flight, a few minutes spent in rebooting the crew's life support system may have unintended and unacceptable consequences. Systems like this have to be ultra-reliable, even if some of their software parts are not. The wise thing to assume in these cases is that no software part is fail-proof, not even those that have been verified exhaustively.

\subsection{Simplicity and Redundancy}

There are two primary strategies for achieving system reliability. The first strategy is to use a design that emphasizes simplicity and robustness. A simple design is easier to understand, easier to test or verify, and easier to operate. The second strategy is to exploit redundancy. If the probability of failure of individual components is statistically independent, the chance of having both a prime and a backup component fail at the 
same time can be made very small. If, for instance, all components have the same probability $p$ of failure, then the probability that all $N$ components fail in an $N$ redundant system would be $p^{N}$. In a nutshell, simplicity seeks to reduce the value of $p$, while redundancy seeks to increase the value of $N$. Trivially, for all values of $N \geq 1$ and $0<\mathrm{p}<1$ both techniques can lower the probability of failure $p^{N}$ for the system.

Unfortunately, one of the basic premises used in the redundancy argument that we used above, the statistical independence of the failure probabilities of components, can be very hard to achieve for software. Well-known are the experiments performed in the eighties by Knight and Leveson with $N$-version programming techniques, which demonstrated that different programming teams tend to make the same types of design errors when working from a common set of (often flawed) design requirements. [KL86] Independently, Sha also pointed out that a decision to apply $N$-version programming cannot be made independently of budget and schedule decisions. With a fixed budget, each of $N$ independent development efforts will inevitably receive only $1 / N$-th of the total project resources. If we compare the expected reliability of $N$ development efforts, each pursued with $1 / N$-th of the project resources, with one targeted effort that can consume all available resources, the tradeoffs become very different. [S01]

Redundancy in the traditional sense, in the way that has proven to work well with hardware systems, therefore cannot be duplicated easily in software systems. By combining the strategies of simplicity and redundancy in a different way, though, we may be able to build larger software systems that are indeed significantly more reliable than any of their individual parts.

\section{Software Architectures for Fault Containment}

Consider a standard software architecture consisting of software modules with welldefined interfaces. Each module performs a separate function. The modules are defined in such a way that information flow across module boundaries is minimized. We will assume here, primarily for simplicity but without loss of generality, that the only way for modules to interact is through message passing over trusted channels. Modules execute (at least logically) on independent hardware, to secure that the crash of one module cannot affect other modules in any other way than across its module interface. A failed module may stop responding, or fail to comply with the interface protocols by sending erroneous requests or responses. We will make a further assumption that module failures can be detected either through consistency checks that are performed inside a module, or by peer modules that check the validity of messages that cross module boundaries.

One could make the argument that a failure that cannot be detected at runtime is not a failure that can be remedied. We will have to accept that not all conceivable types of failures can be defended against with this or any other fault containment discipline. We restrict our attention to those cases where a remedy is at least in principle possible.

In our proposed software architecture, each software module is provided with a simplified backup. During normal system operations, this backup module is idle. When a fault is detected, though, the faulty module is switched offline and the backup 
module replaces it. (Naturally, the backup module can have its own backup, and so on, but we will not pursue this generalization here.) The backup, due to the fact that it is a simplified version of the prime module, may offer fewer services, or it may offer them less efficiently. The purpose of the backup, though, is to provide a survival and recovery option to a partially failed system. It should provide the minimally necessary functionality that is required to "stay alive."

Note that in a traditional system the failing module is its own backup. Upon a failure one simply restarts the module that failed and hopes that the cause for failure was transient. We suggest that we can defend against a substantially larger class of defects if the backup module is distinct from the primary module and deliberately constructed to be significantly simpler than the primary module.

As indicated earlier, if the primary and backup modules are constructed within an $\mathrm{N}$-version programming paradigm, we do not necessarily gain additional reliability from this type of system structure. This system structure will not adequately defend against design and coding errors. Some of the same design errors may be made in the construction of both modules, and if the two modules are of similar size and complexity, they should be expected to contain a similar number of residual coding defects (i.e., coding defects that escape code testing and verification). Our proposal is therefore to make the backup modules significantly simpler than the primary modules.

\subsection{Simplified Redundancy}

The backup modules in our proposed architecture are constructed as simplified versions of the primary modules. Specifically, these backup modules can be designed and build by the same developer(s) that design and build the primary modules. The primary module is build for performance; the backup module is build for correctness. The main purpose for a system architecture of this type is that the backup modules are easier to verify thoroughly. The statistically expected number of residual defects in a backup module should be significantly lower than that of the primary module, if they contain significantly less code that can be checked thoroughly.

The backup module is used to guarantee continuity of operation, though in a possibly degraded state of operation (e.g., slower and likely with reduced functionality). The backup gives the system the opportunity to recover from unexpected failures: the primary module is offline and can be diagnosed and possibly restarted, while the backup module takes care of the most urgent of tasks in the most basic of ways. If code is developed in a hierarchical fashion, using a standardized software refinement approach, the backup module could encapsulate an earlier level in the refinement of the final module: a simpler version of the code that is not yet burdened with all features, extensions, and optimizations that will support the final version, but that does perform the most critical and basic duties in the most straightforward way.

If this approach can be made to work (at the time of writing we not yet completed a realistic case study) we would expect the backup modules to be significantly smaller in size (e.g., in lines of code) than the primary modules. By virtue of being smaller and simpler, the expected number of residual defects in this code should also be smaller. We will tacitly assume here that the number of design and coding defects is proportional to the size of a module, just like the number of syntax and grammar 
mistakes in English prose is proportional to the length of that prose. If the primary module has a probability of failure due to residual defects $p$ and for the backup module the probability of failure is $q$, we would expect to have $1>p>q>0$ (ignoring the boundary cases where we have either certainty of failure or absolute perfection). Because the backup module contains less code, and implements less functionality, it offers fewer opportunities for design and coding defects. The module with its backup now fails with probability $(p . q)$ which should be smaller than the probability $p$ for the same module without the backup.

\subsection{Fault Detection and Secure Fall-Back}

We have assumed that we can tell, in a sufficient number of cases, when a software module fails to perform its intended function due to a design or coding error. There are several ways in which this could work, at least in principle, but none are truly satisfactory. The module code can contain assertions that check for the validity of inputs and outputs (standard pre and post-condition checks), and they can verify that essential invariants are maintained in the module code. But if we assume that the nature of the residual software defects is unpredictable and to first approximation will exhibit itself as a random divergence of the intended or desired behavior, the conclusion will be inevitable that a module cannot reliably detect all occurrences of defects in its own code. Modules can, however, be reasonably expected to check each other. If a module, for instance, detects that faulty input is provided to it across its module interface, the module could declare the peer module that provided the input to be faulty, reject the input, and command the suspect module to be switched over to its backup. There is a close correspondence here to security related problems in mainstream software design: how can a module trust that its peer is reliable? [R98, W89]

There is also another problem that has to be addressed. Even supposing that we would have, or will be able to develop, a reliable defect detection discipline, how precisely can we arrange things in such a way that the switch-over from a primary module to its backup (or vice versa) does not itself introduce a system failure? cf. [AB85, RL81] We do not have answers to these questions, but suggest them as a potentially fruitful area of research in reliable software systems design.

\section{Synopsis}

The goal of this position paper is to suggest that to achieve software reliability we should not restrict ourselves solely to the investigation of ways to achieve zero-defect code, but also more broadly to new methods to produce fail-proof systems. We would like to develop the art of building reliable systems from unreliable parts into a mature software engineering discipline.

The principal method of structuring code we propose to investigate is fairly simple. The code is structured into modules that can fail largely independently. Modules communicate only via well-defined interfaces. Each module is provided with at least one backup that can take over basic operations when the primary module fails. The backup module is constructed to be significantly simpler, smaller, and more reliable 
than the primary that it supports, possibly performing less efficiently and providing less functionality.

This basic mode of operation is already used today in the hardware design of spacecraft. Spacecraft typically do not just have redundant components on board, but also components of different types and designs, providing different grades of service. Most spacecraft, for instance, have both a high-gain and a low-gain antenna. When the high-gain antenna becomes unusable, the more reliable low-gain antenna is used, be it at a significantly reduced bit-rate. Perhaps not surprisingly, this same principle has also been applied on a modest scale in the design of mission critical software, though not always systematically. The MER rover software, for instance, was designed to support two main modes of operations: the fully functional mode with all its features and functions enabled and a minimal basic mode of operation that has been referred to as the "crippled mode." It was precisely this "crippled mode" that made it possible for the software engineers to recover from a serious software anomaly that struck one of the rovers early in its mission. [RN05] Our proposal is to use these principles more systematically, throughout the design of safety and mission critical software components.

\section{Acknowledgement}

The work described in this paper was carried out at the Jet Propulsion Laboratory, California Institute of Technology, under a contract with the National Aeronautics and Space Administration. Specifically, this work is part of a NASA funded project SISM-160, titled Reliable Software Systems Development (RSSD), which targets the development of new tool-based methodologies for reliable software development.

\section{References}

[AB85] Anderson, T., Barrett, P.A., Halliwell, D.N., Moudling, M.L.: An evaluation of software fault tolerance in a practical system. In: Proc. Fault Tolerant Computing Symposium, pp. 140-145 (1985)

[KL86] Knight, J.C., Leveson, N.G.: An Experimental Evaluation of the Assumption of Independence in Multi-version Programming. IEEE Transactions on SoftwareEngineering SE-12(1), 96-109 (1986)

[RN05] Reeves, G., Neilson, T.: The Mars Rover Spirit Flash Anomaly. In: IEEE Aerospace Conference, Big Sky, MT (March, 2005)

[RL81] Rasmussen, R.D., Litty, E.C.: A Voyager attitude control perspective on fault toler-ant systems. In: Proc. AIAA Conf. Alburquerque, NM, August 1981, pp. 241-248 (1981)

[R98] Rushby, J.: Partitioning in Avionics Architectures: Requirements, Mechanisms, and Assurance. Draft technical report, Computer Science Laboratory, SRI (1998)

[S01] Sha, L.: Using Simplicity to Control Complexity. IEEE Software, 20-28 (July-August, 2001)

[W89] Weber, D.G.: Formal specification of fault-tolerance and its relation to computer security. In: Proc. 5th Int. Workshop on Software Spec. and Design, Pittsburgh, PA, May 1989, pp. 273-277 (1989) 


\section{A Discussion on Gerard Holzmann's Presentation}

\section{John Rushby}

I am generally supportive of your proposal, but there is a lot of data from aircraft and rather less so from spacecraft. But most of the serious faults with redundancy management is to say: "When the staff switches over from primaries to backups..." So, aren't you potentially introducing the most dangerous of all mechanisms into your software system?

\section{Gerard Holzmann}

Yes, this is an excellent question. This was one of the bullets on my details. The one thing that I can say about it is that redundancy on spacecraft, currently-I do not know about aircraft and so on, but for spacecraft, I know very precisely-is only used for hardware. So, you can provide a redundant main engine or a redundant antenna. It is never used for software. So this is a different way to apply this technique, and the fact that the redundancy is highly simplified and thoroughly checkable is essential to this. So now, all those issues that you mentioned: "How do you make sure that you do not accidentally switch off for the wrong reason?" all those are valid. But if you do, then basically, you do not suffer major consequences, where normally, in a traditional redundant system, you might.

\section{John Rushby}

Be sure that your state is synchronized, for example. So, the backup has got the same state as the primary, otherwise, you get a [missing word].

\section{Gerard Holzmann}

Yes, so the "hand off" policy is one of those issues about how we do restore states, and that are methods for achieving a [missing word]. So, there is a demo, which I am not going to show now but can show you afterwards, that shows how you can do some of these "hand off" policies.

\section{Tony Hoare}

Thank you for a very interesting talk, and congratulations on your appointment as an engineer. We could have easily seen that you are an engineer by the contents of your talk, of course. It is a lovely example, because the only way to have more faith in your backup than your main source is by proving it. Testing it gives you no more confidence than testing the original. So, it is either prove or nothing. Wonderful! Thank you!

\section{Patrick Cousot}

The architecture that you describe, more or less exists already in aircraft, and it works, but it has a huge cost, and in particular a huge testing cost, because you have to test 
all the combinations of all the generated modes. Because you do less in those simplify things, it may interact with things that do more or less, and these interactions have to be checked, and this is extremely costly. You will pay by a factor of ten for coding and about factor 1,000 for testing. So, what are you going to do about that?

\section{Gerard Holzmann}

Prove you wrong? 